\title{
Pattern-directed attention in uncertain-frequency detection
}

\author{
JAMES H. HOWARD, JR., ALICE J. O'TOOLE, RAJA PARASURAMAN, \\ and KEVIN B. BENNETT \\ The Catholic University of America, Washington, D.C.
}

\begin{abstract}
The role of early pattern components as cues in uncertain frequency detection was investigated in four probe-signal experiments. Listeners heard two consecutive presentations of one of two 12-tone patterns in a noise background. One presentation of the pattern was complete, whereas the other was missing the 11 th (primary) tone. Listeners were required to indicate which presentation was complete. On $20 \%$ of the test trials, the 11 th component of the complete pattern was replaced with one of four probe tones. The results indicated that listeners were more sensitive to the primary tone than to probe tones, and this selective sensitivity changed on a trial-by-trial basis as a function of the attentional cues provided by early pattern components. The data suggested two cue functions: (1) an "informational" function in providing information regarding which of two primary tones is likely to occur on a given trial, and (2) a "frequency" function that "automatically" directs listening to an appropriate frequency range and narrows, or "fine tunes," the listening band.
\end{abstract}

In a typical psychoacoustic detection task, listeners are required to listen for a pure tone in a background of noise. In the popular two-alternative forcedchoice procedure, a tone of known frequency occurs in one of two brief, clearly demarked listening intervals on each trial. One variation of this "standard" procedure involves selecting the test tone from one of two or more frequencies that may or may not be known to the listener (Creelman, 1960; Tanner \& Norman, 1954). This procedure introduces uncertainty regarding the tonal frequency that will occur on any given trial. The results of a number of these uncertain frequency experiments have revealed a small, but consistent, degradation of detection performance when compared with the standard or certain-frequency task (Swets, 1963).

Most explanations of this finding assume that listeners have a limited-bandwidth listening, or attention, band. In the certain-frequency case, this band can be adjusted to match the known frequency of the test signal, whereas in the uncertain-frequency case an optimal location for the listening band cannot be predicted for any given trial. The concept of an attentional band is related to Fletcher's (1940) classic notion of the critical band, but with an emphasis on central, rather than peripheral, factors in audition. As Swets and Kristofferson (1970) have pointed out,

This research was supported by a contract from the Engineering Psychology Group of the Office of Naval Research to the first author. The authors thank Darlene V. Howard, John J. O'Hare, David M. Green, and an anonymous reviewer for their comments on an earlier version of the manuscript. Requests for reprints should be addressed to James H. Howard, Jr., Human Performance Laboratory, The Catholic University of America, Washington, DC 20064. attentional studies generally "reflect the view that frequency selectivity is substantially under the intelligent control of the observer-their focus is on attention rather than on the basilar membrane" (p. 350). The present study reflects this emphasis. Four experiments, which investigate the cues listeners use to adjust their attentional band on a trial-by-trial basis to detect selected individual tones embedded within multitone patterns, are reported.

\section{Uncertain Frequency Experiments}

In an early experiment, Tanner and Norman (1954) employed a four-interval forced-choice method and found that performance fell to chance levels when they shifted the test signal from a known frequency to another frequency without informing the listeners. Greenberg and Larkin (1968) introduced a related probe-signal method in which listeners first learned to detect signals of a single primary frequency in a standard detection task. After this, other signals of different probe frequencies were presented on a small proportion of the trials (e.g., 20\%). Their findings, similar to those of Tanner and Norman (1954), revealed that detection of unexpected probe frequencies that differed from the primary frequency by more than approximately $150-200 \mathrm{~Hz}$ was at a chance level. Greenberg and Larkin argued that the probe-signal method might be used to estimate the shape and width of the listening band; however, others have suggested that the method might be limited for characterizing the precise shape of the auditory filter (Patterson \& Nimmo-Smith, 1980). Macmillan and Schwartz (1975) have used the probe-signal method successfully to demonstrate concurrent two-channel listening in uncertain frequency detection. Other 
studies have demonstrated that uncertain frequency will lead to degraded performance when compared with a single frequency condition, even if the listeners are aware that more than one signal can occur (Creelman, 1960; Green, 1961).

\section{Theories of Uncertain Frequency Detection}

Most theoretical accounts of the uncertain-frequency effect have assumed that listeners use either single or multiple listening bands. The single-band approach, introduced by Tanner, Swets, and Green (1956, as cited in Swets, 1963'), assumes that listeners employ a single listening band that must include the test signal frequency for detection to occur. In an uncertain frequency experiment, the listening band must be swept across a range of frequencies or between two known frequencies and consequently signals will be missed.

On the-other hand, the multiple-band approach, introduced by Green (1958) and modified by Creelman (1960), assumes that listeners base their decisions on the combined outcomes of several listening bands positioned at different frequencies. Different methods are assumed for combining the multiple-filter outputs, but all these models assume that the detectability of an individual tone will decrease as the number of listening bands increases.

Empirical evidence has been reported to support both the single-band (Swets \& Sewall, 1961; Swets, Shipley, McKey, \& Green, 1959) and the multipleband approaches (Creelman, 1960; Green, 1961; Macmillan \& Schwartz, 1975). In general, the two views are difficult to distinguish empirically, and Green (1961; Green \& Weber, 1980) has argued that uncertain frequency effects are generally smaller than would be predicted by either theory. According to Green (1961), this latter finding may simply reflect the fact that considerable subjective uncertainty exists in even the standard detection task, thereby lowering performance relative to a true certainfrequency condition. Other evidence suggests that either a single-or a multiple-band listening strategy may be used, depending on the task context and the individual observer (Green, 1961; Swets, 1963).

In a more recent paper, Johnson and Hafter (1980) proposed an extension of the traditional multipleband model. Their model assumes that two factors are under listener control and can influence performance: (1) the number of listening bands, and (2) the bandwidth(s) of the listening band(s). The first factor is influenced by the listener's expectations regarding the number and frequency of signals likely to occur, whereas the second factor is influenced by the accuracy of the listener's knowledge regarding the test frequencies. Overall, the listener's attentional strategy determines the tradeoff that will occur between the two factors. This model incorporates earlier ideas regarding adjustable filter bandwidth (Sorkin,
Pastore, \& Gilliom, 1968; Swets, 1963), and reflects properties of both the single- and the multiple-band models. Listeners may choose to monitor only a single narrow band under conditions of low-frequency uncertainty or one or more broad bands when signal uncertainty is high.

\section{Control of Attention in Auditory Detection}

In many uncertain-frequency detection studies, the listener's attention is influenced by overall properties of the signal probability distribution (Swets, 1984). In other words, listeners tend to listen for highprobability signals rather than for low-probability signals. Other studies have used explicit signal cues to provide information regarding the likely signal frequency (Gilliom \& Mills, 1976; Kinchla, 1973; Swets \& Sewall, 1961). For example, several studies have demonstrated improved performance under uncertain-frequency conditions when a tone of the same frequency as the test tone preceded the listening interval (Gilliom \& Mills, 1976; Johnson \& Hafter, 1980; Swets \& Sewall, 1961). Cues of this sort have been termed frequency cues (Gilliom \& Mills, 1976), since the cue itself can serve as a frequency model. On the other hand, frequency cues also serve as information cues, since they inform the listener of the likely frequency of the test tone. Swets and Sewall (1961) have shown that pure informational cues (lights) can be as effective as tonal frequency cues in enhancing performance in uncertain-frequency tasks. The results of these cue studies suggest that listeners are able to adopt a listening strategy that permits trial-by-trial variations in their attentional focus.

\section{The Present Study}

The present study investigates the potential frequency and informational cue value of early components in simple tonal patterns when listeners are required to detect the presence of later elements. Watson and his associates have shown in a series of same-different pattern-discrimination experiments that listeners are better able to resolve individual pattern elements under conditions of low stimulus uncertainty than under high-uncertainty conditions (Watson \& Kelly, 1981). Uncertainty was reduced in their experiments by using either highly familiar tonal patterns or by using test components of predictable frequency. Jones, Boltz, and Kidd (1982) have also shown that both the melodic (frequency) and rhythmic (temporal) properties of simple patterns can influence a listener's attention to embedded pattern components. These findings suggest that early pattern components will serve as significant attentional cues for detecting subsequent pattern components.

Four experiments that examine this possibility are reported. The experiments employed a method that combined the traditional probe-signal uncertain frequency and the multielement pattern discrimination 
methods. In each experiment, listeners were presented with pairs of 12 -element tonal patterns. On each trial, one of two patterns was complete and the other had a gap in place of the 11th component. Listeners were required to report which of the two was complete. After training, the high-probability or primary signal in the complete pattern was replaced by one of several probe frequencies on a small percentage of the trials $(20 \%)$. This method paralleled the probe-signal experiments summarized previously, but with the to-be-detected tones occurring within a pattern context rather than in isolation. The primary objective of the present study was to investigate the influence of the 10 initial pattern elements on listeners' ability to detect the test tone in the 11 th position. In particular, conditions were examined in which the early components either provided informational cues only or both informational and frequency cues for the test signals. Since one of two different patterns could occur on any trial, the utility of the first 10 components in directing the listener's attention to the proper test frequency could be determined.

In the first experiment, high- and low-frequency "patterns" of a single repeating frequency were used so that early pattern components could serve as same-frequency attentional cues for the detection of a later element. The early components in this experiment served both as information cues, since the early tones were perfectly correlated with the test frequency for that pattern, and as frequency cues, since they have the same frequency as the test element. In the second experiment, patterns of increasing and decreasing frequencies were presented. Early components of these patterns served primarily as informational cues rather than frequency cues in directing individuals to listen at the appropriate frequency. Experiments 3 and 4 used patterns of rising and falling frequency as in Experiment 2, but with an "offpattern" high-probability primary signal. In these experiments, the pattern context provided an inappropriate frequency cue for the primary test signal. In Experiment 3, listeners had to ignore the pattern context to focus on a single primary frequency common to both patterns, whereas in Experiment 4 the early pattern components could be used only as informational cues to attend selectively to that appropriate frequency on a trial-by-trial basis.

\section{EXPERIMENT 1}

\section{Method}

Particlpants. Four student and staff volunteers between the ages of 20 and 35 years participated in the experiment. None reported any history of hearing disorders.

Stimull. The stimuli consisted of two 12-tone patterns of pure tones into which a test tone would be inserted. The pattern and test tones were selected from a set of five pure-tone signals with frequencies of $700,1150,1600,2050$, and $2500 \mathrm{~Hz}$. The tones were
$100 \mathrm{msec}$ in duration with 5 -msec onset/offset ramps to eliminate audible clicks. The energy levels of the five tones were adjusted to yield equally detectable signal-to-noise ratios using the following equation from Green, McKey, and Licklider (1959): $10 \log (\mathrm{E} / \mathrm{No})$ $=2(f / f c)+16.6 \mathrm{~dB}$, where $E=$ signal energy, No= noise power density, $f=$ signal frequency, and $\mathrm{fc}=1000 \mathrm{~Hz}$. With this adjustment, the signal presentation levels ranged from approximately $63.6 \mathrm{~dB} \mathrm{SPL}(700 \mathrm{~Hz})$ to approximately $67.2 \mathrm{~dB} \mathrm{SPL}(2500 \mathrm{~Hz})$ in equal steps of $.9 \mathrm{~dB}$. The tones were presented in a continuous noise background $(400-3500 \mathrm{~Hz})$.

The two constant patterns were constructed of 12 repetitions of either the $1150-\mathrm{Hz}$ tone (low pattern) or the $2050-\mathrm{Hz}$ tone (high pattern) with an intertone interval of 20 msec. The $1150(2050)-\mathrm{Hz}$ tone was the high-probability primary test signal for the low(high) constant pattern and the remaining four tones were the lowprobability probe test signals. The two patterns and the five test frequencies used in this experiment are shown schematically in Figure 1a. On every trial, either the high- or low-frequency pattern was presented twice, once as a complete pattern and once as an incomplete pattern. The incomplete pattern had a $100-\mathrm{msec}$ delay substituted for the 11 th tone presentation, whereas the complete pattern had either the primary or a probe signal at this test position. On the practice trials, only the primary tone occurred at the test position (i.e., $1150 \mathrm{~Hz}$ for the low pattern and $2050 \mathrm{~Hz}$ for the high pattern). The practice trials began with a signal-tonoise ratio of $28.6 \mathrm{~dB}(1000 \mathrm{~Hz})$, which was reduced gradually to the test level of $18.6 \mathrm{~dB}(1000 \mathrm{~Hz})$. During the two test blocks, the 11 th pattern position contained the primary tone on $80 \%$ of the trials and a probe tone on the remaining $20 \%$ of the trials. Since there were 160 test trials in a test block, each of the four probes for each pattern occurred on eight trials, whereas the primary occurred on 128 trials in each test block.

Apparatus. All experimental events were controlled by a generalpurpose laboratory computer. The tones were synthesized using standard digital techniques. They were output on a 12-bit digitalto-analog converter at a sampling rate of $10 \mathrm{kHz}$, low-pass filtered at $4 \mathrm{kHz}$ (Khron-Hite Model 3550), attenuated (Charybdis programmable attenuator), and presented monaurally (right ear) over a calibrated TDH -49 headphone with an MX-41/AR cushion. The noise was produced by a broadband generator (Bruel \& Kjaer Model 1402), bandpass filtered (Khron-Hite Model 3750) and mixed with the tones using a laboratory-constructed passive mixer. Verbal prompts were presented on a video monitor in the doublewalled testing booth (Industrial Acoustics), and a solid-state keyboard was used for listener responses.

Procedure. Listeners were tested individually over $\mathbf{S}$ days. A twoalternative forced-choice task was used on each day. On the first day, listeners were told that they would be hearing pairs of simple patterns on each of a series of trials. One of these patterns would be complete, whereas the other would have a single component tone missing. They were told that their task would be to determine which of these otherwise identical patterns was complete. The listeners were also told that the patterns would be played in a steady noise to make the task more difficult; however, to permit familiarization with the task, the patterns would initially be relatively easy to hear.

On each of the $s$ days, the listeners received three blocks of trials, one practice block (100 trials) and two test blocks (160 trials in each). Each listener had 500 practice trials and 1,600 test trials in all. The order of presentation was determined randomly, both within (order of complete and incomplete patterns) and across (pattern and test frequency) trials within each block.

\section{Results and Discussion}

The percentage of correct responses was determined for each test frequency for each pattern and listener in the experiment. Percent correct provides a bias-free estimate of sensitivity because a twoalternative forced-choice procedure was used. The 

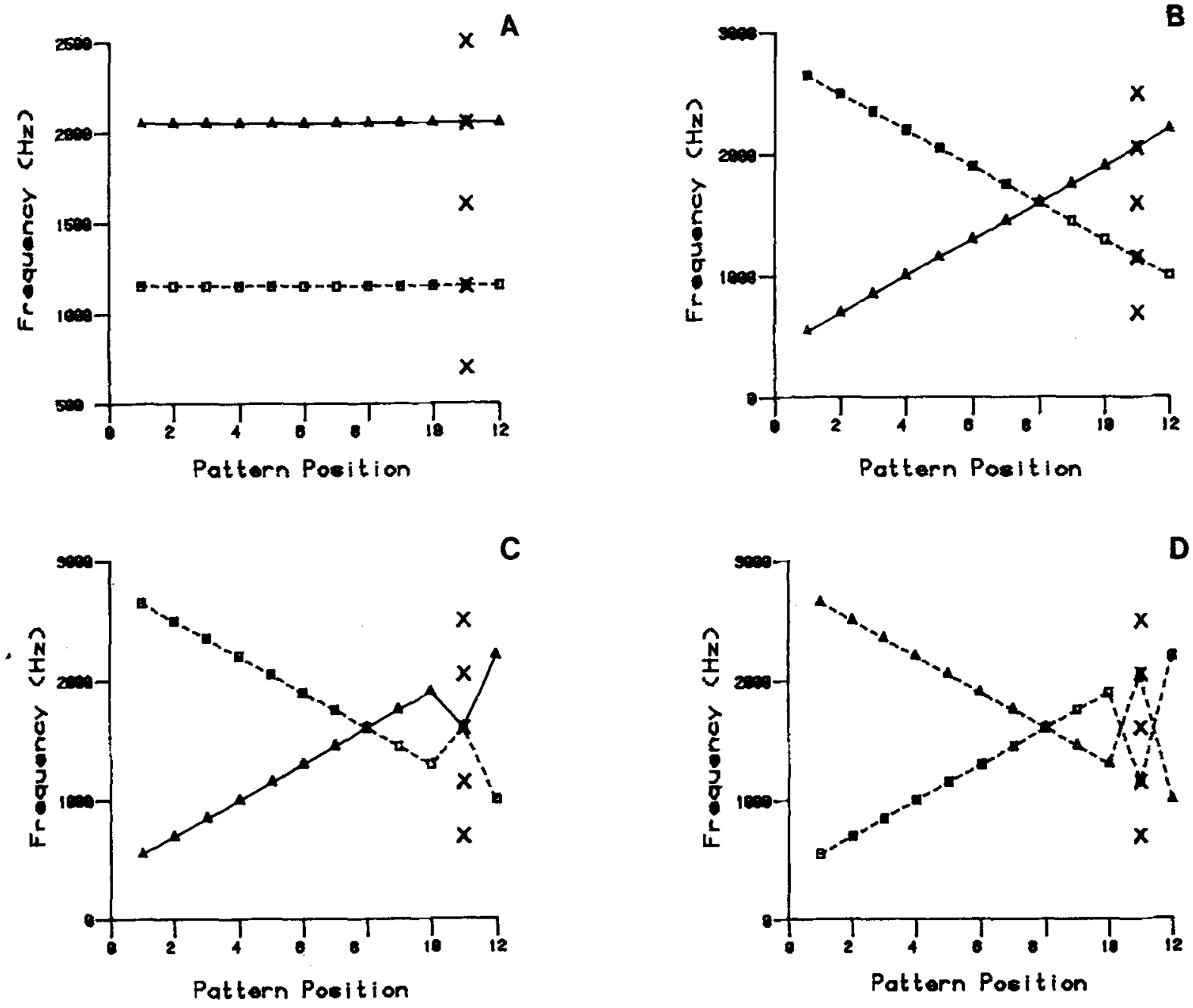

Figure 1. Schematic plots of the stimulus patterns and test signals for Experiments 1 through 4 (plots $A$ through D).

results of this analysis are shown collapsed across the four listeners in Figure 2 for both the high- and lowfrequency patterns. A two-way analysis of variance (pattern $\times$ frequency) with repeated measures on both factors was carried out on these data. This analysis revealed significant main effects of pattern $[F(1,3)$ $=10.86, p<.05]$ and frequency $[F(4,12)=3.55$, $p<$ $.05]$ and a significant pattern $\times$ frequency interaction $[F(4,12)=8.88, p<.01]$. To investigate the interaction further, the simple effects of pattern were determined for each of the five test frequencies. A significant simple effect of pattern was observed at the two primary frequencies of $1150 \mathrm{~Hz}[\mathrm{~F}(1,3)=963.60$, p < $.05]$ and $2050 \mathrm{~Hz}[\mathrm{~F}(1,3)=651.60, \mathrm{p}<.05]$ and at the $2500-\mathrm{Hz}$ probe frequency $[\mathrm{F}(1,3)=836.40, \mathrm{p}<.05]$. The simple effects of pattern were not significant for either the $700-\mathrm{Hz}$ probe $[\mathrm{F}(1,3)<1.0]$ or the $1600-\mathrm{Hz}$ probe $[F(1,3)<1.0]$. As is evident in Figure 2, performance was substantially better on the highprobability primary test signals for each pattern than it was on the low-probability probes. This suggests

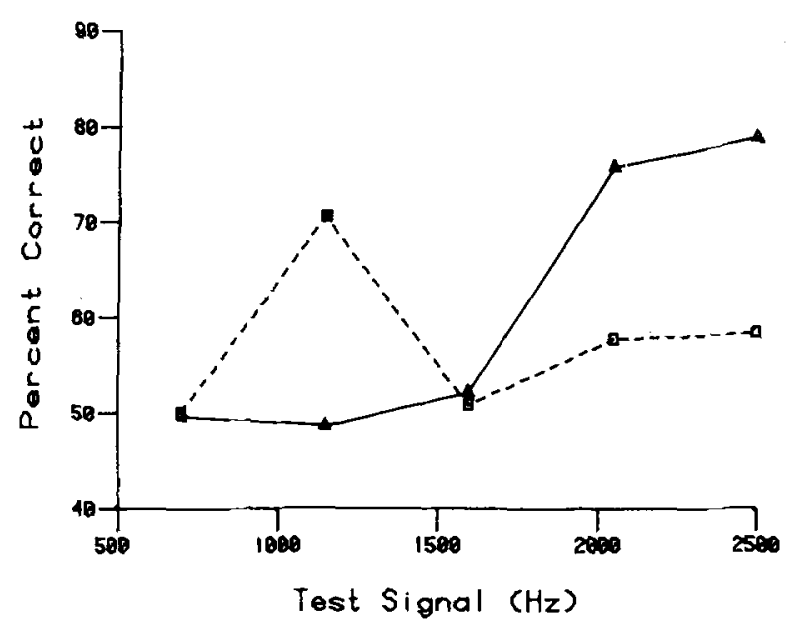

Figure 2. Mean percent correct collapsed across the four listeners of Experiment 1 at each test frequency for both the high (solid line, triangles) and the low (dashed line, squares) patterus. 
that the listeners were able to use the initial segment of the pattern to attend selectively to the likely test frequency for that pattern, and that this selection occurred on a trial-by-trial basis. The only exception to this was on high-pattern trials in which the $2500-\mathrm{Hz}$ probe was highly detectable ( $79 \%$ correct). This unexpected finding suggests that listeners may have used an unusually broad listening band on the highpattern trials, an interpretation consistent with the constant- $Q$ properties assumed for the critical band. This possibility was considered again in Experiment 2.

\section{EXPERIMENT 2}

Overall, the results of the first experiment are consistent with the hypothesis that listeners can use early pattern components as frequency and/or informational cues to shift their listening band to highprobability test frequencies on a trial-by-trial basis. In the second experiment, patterns of rising and falling pitch were used in which the early pattern components were at frequencies either below (rising pattern) or above (falling pattern) the optimal listening frequency. Hence, the early pattern components should serve primarily as informational cues. It was predicted that if pattern-dependent cue effects were obtained in this experiment, then the value of early pattern components as informational cues for trialby-trial selective listening would be established.

\section{Method}

Participants. Four student and staff volunteers between 25 and 33 years of age participated in the experiment. None reported any history of hearing disorders, and none had participated in Experiment 1 .

Stimuli. A set of 15 pure tones ranging in frequency from 550 to $2650 \mathrm{~Hz}$ in equal steps of $150 \mathrm{~Hz}$ (including the five described for Experiment 1) were used to construct two 12-tone patterns. For the rising pattern, the tones between 550 and $2200 \mathrm{~Hz}$ were presented in ascending order, whereas for the falling pattern, the tones between 2650 and $1000 \mathrm{~Hz}$ were presented in descending order. As in the previous experiment, all tones were $100 \mathrm{msec}$ in duration with a 5 -msec onset/offset ramp, and the patterns had an intertone interval of $20 \mathrm{msec}$. The intensity levels of all 15 tones were adjusted to be as detectable in noise as in Experiment 1. The two patterns are shown schematically in Figure $1 \mathrm{~b}$. The primary $(2050 \mathrm{~Hz}$ for the rising pattern and $1150 \mathrm{~Hz}$ for the falling pattern) and probe test signals were presented in the 11 th pattern position and were identical to those used in Experiment 1. The continuous noise background was also identical to that used in Experiment 1.

Apparatus. The apparatus was identical to that used in Experiment 1 .

Procedure. The procedure was the same as in Experiment 1, except that participants were instructed that the patterns would be either rising or falling in pitch.

\section{Results and Discussion}

A bias-free percentage of correct responses was determined for each test frequency for each pattern and listener in the experiment. These data are shown

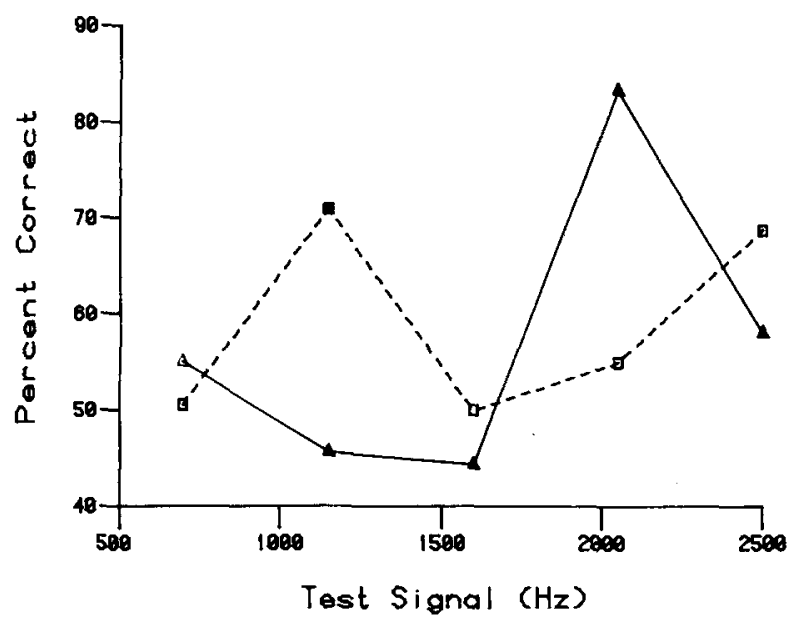

Figure 3. Mean percent correct collapsed across the four listeners of Experiment 2 at each test frequency for both the rising (solid line, triangles) and the falling (dashed line, squares) patterns.

collapsed across the four listeners in Figure 3 for both the rising and falling patterns. A two-way analysis of variance (pattern $\times$ frequency) with repeated measures on both factors was performed on the data. This revealed a significant main effect of frequency $[F(4,12)=3.27, p<.05]$ and a significant pattern $x$ frequency interaction $[F(4,12)=6.45, \mathrm{p}<.01]$. The main effect of pattern was not significant $[F(1,3)<$ 1]. An analysis of the simple effects of pattern at each test frequency revealed significant effects at the $1150-\mathrm{Hz}$ primary $[\mathrm{F}(1,3)=20.71, \mathrm{p}<.01]$ and at the $2050-\mathrm{Hz}$ primary $[\mathrm{F}(1,3)=26.02, \mathrm{p}<.01]$, but no significant simple effect of pattern at any of the probe test frequencies $[700 \mathrm{~Hz}, F(1,3)<1 ; 1600 \mathrm{~Hz}, F(1,3)$ $=1.02$; and $2500 \mathrm{~Hz}, F(1,3)=3.67$, ps $>.05]$.

Inspection of Figure 3 suggests a finding very similar to that observed in the results of Experiment 1. Specifically, the high-probability primary test signals were detected more readily than were the low-probability probes. Furthermore, since the effect was pattern specific, it is clear that listeners were adjusting their listening band to "appropriate" frequencies on a trial-by-trial basis. These findings also demonstrate the informational value of the initial pattern components, since these tones were not at the primary test frequency. If the average performance advantage for the primary test signals over the probe signals is taken as a measure of "cue effectiveness," then the rising and falling patterns used in this experiment (23.6\% primary advantage) were as effective as the constant-frequency patterns used in the first experiment (23.0\% primary advantage).

As in Experiment 1, the highest frequency probe signal $(2500 \mathrm{~Hz})$ was detected more often than any of the other probes $(63.4 \%$ vs. $50.1 \%)$. However, unlike the results of the first experiment, the present results do not reveal a differential sensitivity to this 
probe when the primary is also high-frequency $(2050 \mathrm{~Hz})$. This suggests that any special status attributable to this probe may be due to a very small and theoretically uninteresting overall sensitivity difference that was not eliminated by our efforts to equate sensitivity across frequency (see Method, Experiment 1).

\section{EXPERIMENT 3}

Although the results of Experiment 2 indicate that off-primary frequencies can serve effectively as selection cues for a primary frequency, the monotonicity of the rising and falling patterns may have induced a frequency-directed listening strategy. In other words, in both Experiment 1 and Experiment 2, listeners could simply track the frequencies of the initial pattern components to direct their listening band to the appropriate frequency. Such frequency-directed attention was examined further in Experiment 3. Patterns with initially rising or falling pitch were used as in Experiment 2, but a single high-probability primáry test signal was used at an off-pattern frequency either below the on-pattern frequency for the rising pattern or above it for the falling pattern. Under these conditions, a listener need only listen at the primary frequency on every trial regardless of the pattern context. If the listener's attention is directed automatically to the on-pattern frequency by the rising and falling patterns, then the performance advantage revealed for the primary signal in the first two experiments should not occur. Rather, the results should be similar to those of Experiment 2, with the on-pattern frequencies being most readily detectable. On the other hand, if listeners are able to ignore the pattern context and direct their attention to the constant off-pattern primary frequency, then a performance advantage should be found for the primary frequency.

\footnotetext{
Method

Participants. Six student volunteers between 18 and 22 years of age participated in the experiment. None reported any history of hearing disorders; however, two listeners were dropped from the experiment when they were unable to perform above chance on the preliminary practice trials. None of the participants had served in either of the previous experiments.

Stimuli. Rising and falling patterns were constructed from the tone set used in Experiment 2. For both patterns, however, the $1600-\mathrm{Hz}$ tone was used as the high-probability primary test signal, with the 700-, 1150-, 2050-, and $2500-\mathrm{Hz}$ tones serving as lowprobability test probes. As in the previous experiments, only the primary probes were presented during practice. The stimuli were otherwise identical to those used in Experiment 2. The two stimulus patterns are shown schematically in Figure $1 \mathrm{c}$.

Apparatus. The apparatus was identical to that used in Experiment 1.

Procedure. The procedure was the same as that used in Experiment 1 , except that listeners were told that the patterns would be either generally rising or generally falling in pitch.
}

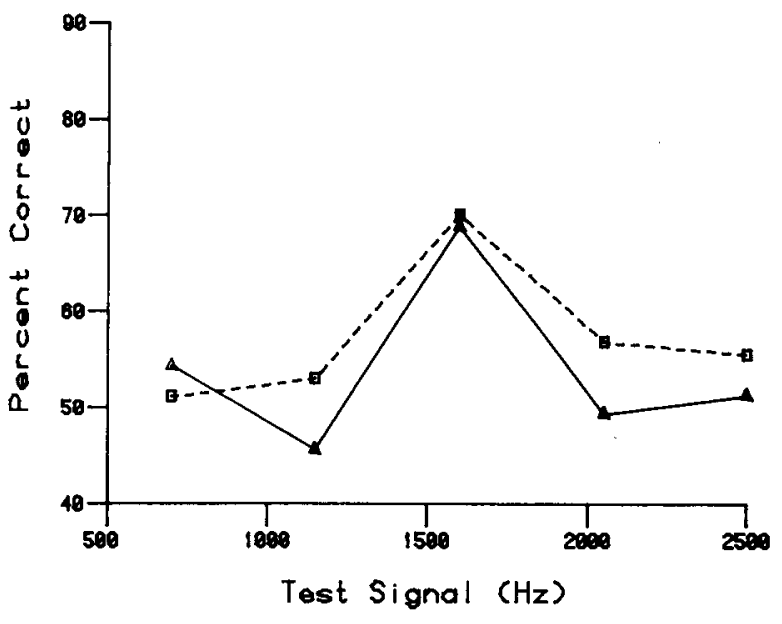

Figure 4. Mean percent correct collapsed across the four listeners of Experiment 3 at each test frequency for both the rising (solid line, triangles) and the falling (dashed line, squares) patterns.

\section{Results and Discussion}

A bias-free percentage of correct responses was determined for each test frequency for each pattern and listener in the experiment. These data are shown collapsed across the four listeners in Figure 4 for both the rising and falling patterns. A two-way analysis of variance (pattern $\times$ frequency) with repeated measures on both factors revealed a significant main effect of frequency $[F(4,12)=7.59, p<.01]$. Neither the main effect of pattern $[F(1,3)=1.10]$ nor the pattern $\times$ frequency interaction $[F(4,12)<1.0]$ was significant.

As may be seen in Figure 4, the high-probability off-pattern primary was more detectable than the onpattern probes for both the rising and the falling patterns. This indicates that listeners were able to center their listening bands at the off-pattern primary frequency despite the conflicting frequency context provided by the patterns. Nevertheless, the average performance advantage for the primary over the probe signals $(17.2 \%)$ was somewhat smaller in this experiment than in the first two experiments $(23.0 \%$ and $23.6 \%$, respectively). Although not definitive, this suggests that the pattern context in the present experiment may have disrupted the listeners' attentional strategy. Automatic, frequency-directed listening seems unlikely, however, since the rising and falling patterns did not influence sensitivity to the onpattern probes differentially.

\section{EXPERIMENT 4}

The results of Experiment 3 revealed that listeners generally are able to dismiss a pattern-frequency context when it is an inappropriate attentional cue for 
the task. Experiment 4 was designed to investigate the ability of listeners to use early pattern components selectively as informational cues for off-pattern primary test signals. Rising and falling patterns were used as in Experiments 2 and 3. For this experiment, however, the high-probability primary tone for the rising pattern was the on-pattern frequency for the falling pattern, and the primary for the falling pattern was the on-pattern frequency for the rising pattern. If listeners were able to use the early pattern components only as informational cues, then a patternspecific performance advantage should be obtained for the primary frequencies. On the other hand, if frequency-directed listening occurred or the inappropriate pattern context was generally disruptive, as in Experiment 3, then the primary advantage should be absent or reduced in magnitude.

\section{Method}

Participants. Four student volunteers between the ages of 18 and 22 participated in the experiment. None reported any history of hearing disorders, and none had served in any of the previous experiments.

Stimull. Rising and falling patterns were constructed from the tone set used in Experiment 2. For the rising (falling) pattern, however, the $1150(2050)-\mathrm{Hz}$ off-pattern tone was used as the highprobability primary test signal. The low-probability test probes were the $700-, 1600-, 2050$, and $2500-\mathrm{Hz}$ tones for the rising pattern and the $700-, 1150-, 1600-$, and $2500-\mathrm{Hz}$ tones for the falling pattern. These patterns are shown schematically in Figure 1d. As in the previous experiments, only the primary probes were presented during practice.

Apparatus. The apparatus was identical to that used in Experiment 1.

Procedure. The procedure was the same as that used in Experiment 3.

\section{Results and Discussion}

A bias-free percentage of correct responses was determined for each test frequency for each pattern and listener in the experiment. These data are shown collapsed across the four listeners in Figure 5 for both the rising and falling patterns.

Examination of this figure suggests pattern-specific differences in sensitivity. However, the performance advantage obtained $(\mathbf{8 . 6 \% )}$ ) is considerably smaller than that observed in the earlier experiments $(23.0 \%$, $23.6 \%, 17.5 \%$, for Experiments 1, 2, and 3, respectively). A closer examination of these data revealed that, unlike in the earlier experiments, one listener had a pattern of results opposite to that of the other three. This individual showed increased sensitivity to the low-probability, on-pattern probes and chance performance on the high-probability, offpattern primaries. This result suggests that she employed a frequency-directed listening strategy. The data, collapsed across the remaining three consistent. listeners, are shown in Figure 6.

A two-way analysis of variance (pattern $\times$ frequency) with repeated measures on both factors was performed on these data. This analysis showed a sig-

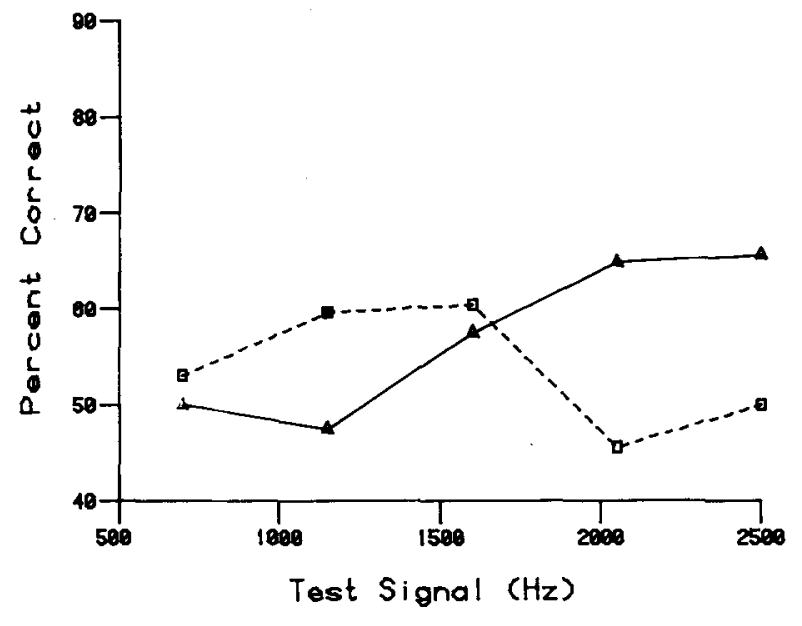

Figure 5. Mean percent correct collapsed across the four listeners of Experiment 4 at each test frequency for both the falling (solid line, triangles) and the rising (dashed line, squares) patterns.

nificant main effect of pattern $[F(1,2)=20.57$, $p<$ $.05]$ and a significant pattern $\times$ frequency interaction $[F(4,8)=12.70, p<.01]$. No reliable main effect of pattern $[F(4,8)=1.92, p>.05]$ occurred. An analysis of the simple effects of pattern at each test frequency revealed significant differences at the two primary frequencies [1150- $\mathrm{Hz}$ primary for the rising pattern, $\mathrm{F}(1,3)=34.72, \mathrm{p}<.01 ; 2050-\mathrm{Hz}$ primary for the falling pattern, $F(1,3)=90.50, p<.01$, and at the highest probe frequency, $F(1,3)=51.17, \mathrm{p}<.01]$. The pattern effect did not approach significance at either of the other probe frequencies [700 and $1600 \mathrm{~Hz}$, $F(1,3)<1.0]$.

As may be seen in Figure 6, sensitivity was greater for the high probability, but off-pattern, primary for

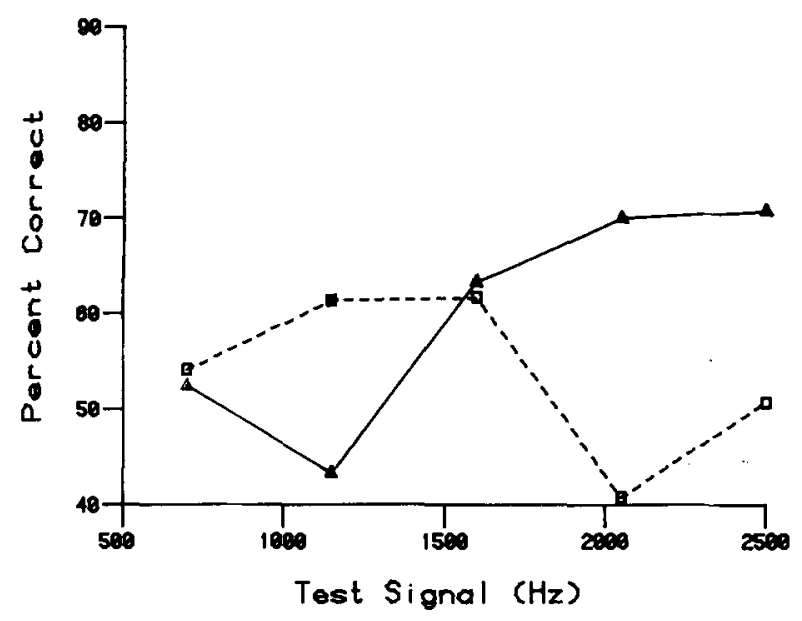

Figure 6. Mean percent correct collapsed across the three consistent listeners of Experiment 4 at each test frequency for both the falling (solid line, triangles) and the rising (dashed line, squares) patterns. 
both the rising $(1150 \mathrm{~Hz})$ and the falling $(2050 \mathrm{~Hz})$ patterns. This indicates that the listeners were able to use the early pattern components as informational cues despite the misleading frequency direction of the pattern. Nevertheless, the cue advantage that occurred was weaker $(11.1 \%)$ than in the first three experiments $(23.0 \%, 23.6 \%$, and $17.2 \%$, respectively), suggesting that the frequency context provided by the early pattern components was not ignored totally. This is consistent with the results of Experiment 3, in which an inappropriate frequency context disrupted single-frequency listening. In the present experiment, disruption was greater for either or both of two reasons: (1) Unlike Experiment 3, the present experiment required trial-by-trial attentional shifting, and (2) the high-probability primaries were further off the frequency context (differences of 900 vs. $450 \mathrm{~Hz}$ ) of the pattern in the present experiment than in Experiment 3 . The greater sensitivity observed for the $2500-\mathrm{Hz}$ probe during the high-frequency listening is consistent with the results of Experiment 1. This finding, together with the relatively high sensitivity to the central $1600-\mathrm{Hz}$ probe for both patterns $(62.5 \%)$, suggests that listeners were using a broad listening band in the present experiment. Overall, then, it appears that although most listeners were able to use early pattern components as informational cues, the misleading frequency context provided by these tones led to a very poor tuning of the attentional filter. The inability of one listener to avoid on-pattern listening further illustrates this point.

\section{GENERAL DISCUSSION}

Overall, the results of this study support several conclusions regarding the role of early pattern components as cues in uncertain-frequency detection. First, the results of Experiments 1, 2, and 4 support the conclusion of earlier studies that listeners can shift their attention to likely frequencies on a trialby-trial basis. Second, it is clear from these experiments that early pattern components can serve as cues to reduce uncertainty for the detection of later pattern elements.

Third, the findings also suggest that it is necessary to distinguish two functions for listening cues in this context. On the one hand, the cues in the three uncertain-frequency experiments $(1,2$, and 4$)$ served an informational function to reduce uncertainty regarding the likely test frequency on any trial. On the other hand, Experiments 3 and 4 revealed a distinct frequency cuing function of the early pattern cues. In the certain-frequency task used in Experiment 3, the rising and falling pattern contexts provided an inappropriate frequency cue which degraded listening performance. Furthermore, in Experiment 4, in which only the informational content of early pattern components was relevant for selective listening, the conflicting frequency cues provided by these components were also disruptive. One of the four listeners in this experiment ignored the informational content of the early components entirely and listened at the inappropriate on-pattern frequencies cued by the rising and falling tonal patterns. The remaining listeners were able to use the informational cues, but with only a poorly tuned, broad listening band.

Fourth, the results of both Experiments 3 and 4 suggest that listeners are unable to ignore completely the frequency content of cues even when the signal probabilities make it inappropriate as a cue. This automatic processing of frequency information is also supported by the results of a sequential analysis carried out on uncertain-frequency-detection data by Swets et al. (1959). This analysis revealed a greater sensitivity to signals that were detected correctly on the previous trial, suggesting that recently experienced tones serve as implicit frequency cues. A similar result was found in a sequential analysis carried out on the data of the three uncertain-frequency experiments in the present study. In all three experiments, listeners were more likely to detect tones that were heard on the previous trial than those that were missed on the previous trial $(.74$ vs. $.71, .75$ vs. .69 , and .64 vs. .60 for Experiments 1, 2, and 4, respectively). This is also consistent with the finding of Johnson and Hafter (1980) that tonal cues that matched the test frequency led to better detectability than did cues that did not match the test frequency.

These conclusions may be related to the two-factor theory of uncertain-frequency detection presented by Johnson and Hafter (1980). They argue that two factors-the number of listening bands, and the bandwidth(s) of these band(s)-influence performance in uncertain-frequency detection. It is possible that the informational function of listening cues influences the first of these factors. Specifically, the cue provides information regarding the number and location of bands to be monitored. In contrast, the frequency function of cues may influence the second of these factors. That is, appropriate frequency contexts that either match the test frequency or are directed toward the test frequency allow finer tuning (i.e., a narrower bandwidth) than do inappropriate frequency contexts. Although speculative, without further research, the results of the present study suggest that the number and location of listening bands is under direct listener control, but that the bandwidth factor is influenced by relatively automatic processes which may not be under direct listener control.

\section{REFERENCES}

Creelman, C. D. (1960). Detection of signals of uncertain frequency. Journal of the Acoustical Society of America, 32, 805-810. 
Fletcher, H. (1940). Auditory patterns. Review of Modern Physics, 12, 47-65.

Gilliom, J. D., \& Mills, W. M. (1976). Information extraction from contralateral cues in the detection of signals of uncertain frequency. Journal of the Acoustical Society of America, 59, 1428-1433.

Green, D. M. (1958). Detection of multiple component signals in noise. Journal of the Acoustical Society of America, 30, 904-911.

Green, D. M. (1961). Detection of auditory sinusoids of uncertain frequency. Journal of the Acoustical Society of America, 32, 897-903.

Green, D. M., McKey, M. J., \& Licklider, J. C. (1959). Detection of a pulsed sinusoid in noise as a function of frequency. Journal of the Acoustical Society of America, 31, 1446-1452.

Green, D. M., \& Weben, D. L. (1980). Detection of temporally uncertain signals. Journal of the Acoustical Society of America, 67, 1304-1311.

Graznbeno, G. Z., \& Larin, W. D. (1968). Frequency-response characteristic of auditory observers detecting signals of a single frequency in noise: The probe-signal method. Journal of the Acoustical Society of America, 44, 1513-1523.

Johnson, D. M., \& HAFTER, E. R. (1980). Uncertain-frequency detection: Cuing and condition of observation. Perception \& Psychophysics, 28, 143-149.

Jones, M. R., Boltz, M., \& KIDD, G. (1982). Controlled attending as a function of melodic and temporal context. Perception \& Psychophysics, 32, 211-218.

Kinchla, R. A. (1973). Selective processes in sensory memory: A probe-comparison procedure. In S. Kornblum (Ed.), Attention and performance IV. New York: Academic Press.

Macmillan, N. A., \& Schwartz, M. (1975). A probe-signal investigation of uncertain-frequency detection. Journal of the Acoustical Society of America, 58, 1051-1058.

Patterson, R. D., \& Nimmo-Smith, I. (1980). Off-frequency listening and auditory filter asymmetry. Journal of the Acoustical Society of America, 67, 229-245.
Sorkin, R. D., Pastore, R. E., \& Gilliom, J. D. (1968). Signal probability and the listening band. Perception \& Psychophysics, 4, 10-12.

Swets, J. A. (1963). Central factors in auditory frequency selectivity. Psychological Bulletin, 60, 429-441.

Swets, J. A. (1984). Mathematical models of attention. In R. Parasuraman \& D. R. Davies (Eds.), Varieties of attention. New York: Academic Press.

Swets, J. A., \& Kaistorferson, A. B. (1970). Attention. Annual Review of Psychology, 21, 339-366.

Swets, J. A., \& Sewald, S. T. (1961). Stimulus vs response uncertainty in recognition. Journal of the Acoustical Society of America, 33, 1586-1592.

Swets, J. A., Shipley, E. F., McKey, M. J., \& Green, D. M. (1959). Multiple observations of signals in noise. Journal of the Acoustical Society of America, 31, 514-521.

TANnen, W. P., \& Norman, R. Z. (1954). The human use of information. II: Signal detection for the case of an unknown signal parameter. Transactions of the IRE Professional Group on Information Theory, PGIT 4, 222-227.

Watson, C. S., \& Kelly, W. J. (1981) The role of stimulus uncertainty in the discrimination of auditory patterns. In D. J. Getty \& J. H. Howard, Jr. (Eds.), Auditory and visual pattern recognition. Hillsdale, NJ: Erlbaum.

\section{NOTE}

1. The Tanner, Swets, and Green reference cited by Swets (1963) is as follows: Tanner, W. P., Swets, J. A., \& Green, D. M. (1956). Some general properties of the hearing mechanism. (Tech. Rep. No. 30). Ann Arbor: University of Michigan, Electonic Defense Group.

(Manuscript received August 15, 1983; revision accepted for publication December 27, 1983.) 